\title{
Pregnancy outcomes in different stages of systemic lupus erythematosus among Chinese women - a retrospective cohort study
}

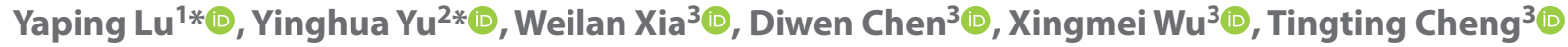 \\ ${ }^{1}$ Department of Obstetrics and Gynecology, Maternal and Child Health Care of Shandong Province, Jinan, China \\ ${ }^{2}$ Department of Obstetrics and Gynecology, Zhongda Hospital Lishui Branch, affiliated to Southeast University, Nanjing, China \\ ${ }^{3}$ Department of Obstetrics and Gynecology, Lishui People's Hospital, affiliated Hospital 6 to Wenzhou Medical University, Lishui, China \\ *contributed equally
}

\begin{abstract}
Objectives: To analyze the outcomes of pregnancies and risk factors in Chinese women with different stages of systemic lupus erythematosus (SLE).

Material and methods: A total of 55 conceptions in 52 patients with SLE between Jan 2007 and Jan 2019 were retrospected systematically from a general hospital graded $3 \mathrm{~A}$ in China. Medical records provided us a good way to retrieve the clinical parameters and lab data of patients.

Results: Pregnant women with SLE activity had significant hyperimmunoglobulin, hypocomplement, low platelet counts, high erythrocyte sedimentation rate, C-reactive protein and 24-h urine protein. Hydroxychloroquine had been used to reduce the rates of SLE activity in pregnant women. Logistic regression analysis showed low platelet counts, hypocomplement and 24-h urine protein were significantly correlated with fetal loss. Compared to those in stable stage, the active SLE patients have more risks of hypertensive disorders of pregnancy, thrombocytopenia, lupus nephritis and placental infarction, and have worse fetal outcomes, including the higher rate of fetal loss, preterm and asphyxia neonatorum.

Conclusions: Different stages of SLE during pregnancy are closely related to maternal and fetal outcomes. It is imperative to provide SLE women with pregnancy consultation and regular multispecialty care.

Key words: systemic lupus erythematosus; clinical parameters; lab data; maternal outcome; fetal outcome
\end{abstract}

Ginekologia Polska 2021; 92, 5: 365-370

\section{INTRODUCTION}

Systemic lupus erythematosus (SLE) is a disease involving autoimmune system, with a morbidity of 4-35 cases per 100 thousand people annually, which preponderantly affects women considerably more often than men, particularly women of reproductive age [1]. As multidisciplinary managements have increasingly involved and the treatments of SLE pregnant women have been improved, the pregnancy outcomes have significantly ameliorated for decades [2]. But such patients are still considered to have high-risk pregnancies. The general trend shows that pregnancy in patients with SLE tend to have higher maternal mortality, exacerbations of disease activity, fewer live births and more complications during pregnancy, including a higher risk of preeclampsia/eclampsia, C-section, prematurity, in- trauterine growth restriction (IUGR), neonatal death and post-partum infection [3-6]. While SLE has many systemic effects, the fertility of these patients is typically unaltered from the general population [3].

Given the high popularity of SLE among women and its damage to maternal and infant health, more researches are needed to have better comprehensions about the impacts of active disease on the safety of mother and fetus as the data remain inconclusive [7]. This study aimed to evaluate factors associated with clinical outcome of active and non-active SLE in Chinese pregnant patients.

\section{MATERIAL AND METHODS}

A retrospective analysis about the medical records of 55 sequential conceptions in 52 patients with SLE in

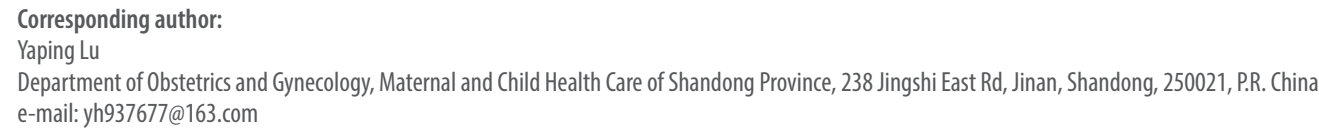


a general hospital graded 3A from Jan 2007 to Jan 2019 was carried out. The diagnosis of SLE is based on the revised criteria formulated by the American College of Rheumatology. Multiple gestations and elective terminations during the first trimester for personal reasons were excluded. This study was authorized from the Ethics Committee and received permission to acquire clinical parameters from the Medical Director affiliated to Lishui People's Hospital. The whole of patient information was kept confidential.

Patients' clinical characteristics were gathered with lab information from medical records. Lab data consisted of hemoglobin (HB), white blood cell (WBC), platelet (PLT), erythrocyte sedimentation rate (ESR), C-reactive protein (CRP), urine protein (PRO), 24-hour urine protein (24-h PRO), albumin (ALB), serum creatinine (CR), blood urea nitrogen (BUN), totalcholesterol (TC), triglyceride (TG), low density lipoprotein cholesterol (LDL-C), high density lipoprotein cholesterol (HDL-C) along with immunologic information including immuneglobulin $A$, immuneglobulin $M$, immuneglobulin G, complement 3 (C3), complement 4 (C4), antinuclear antibodies (ANA), anti-ds DNA antibodies (anti-dsDNA), anti-Smith antibody (anti-Sm), anti-Ro/SSA antibodies, anti-La/SSB antibodies, antiphospholipid antibodies (aPL). All laboratory tests were performed using standardised methods. The placentas were sent for pathological examination after delivery.

The disease activity of SLE was made an appropriate evaluation based on the SLE Disease Activity Index 2000 (SLEDAI-2K) [8]. Patients were categorized into two groups, according to SLEDAI score: active SLE group $(\geq 5)$ and non-active SLE group ( $\leq 4)$.

Maternal outcomes included hypertensive disorders of pregnancy (HDP), gestational diabetes mellitus (GDM), anemia, thrombocytopenia, lupus nephritis, premature rupture of membranes (PROM), oligohydramnios, postpartum haemorrhage (PPH), placental infarction and chorioamnionitis in pathological diagnosis. Pregnancy-induced hypertension (PIH) was a state of blood pressure $\geq 140 / 90 \mathrm{mmHg}$ on two occasions apart at least $\geq 6$ hours observed after the $20^{\text {th }}$ gestational week. Proteinuria $>0.3 \mathrm{~g} / \mathrm{L} /$ day with hypertension was named pre-eclampsia. In this study, PIH and pre-eclampsia were both classified as HDP for a small number of cases. When hemoglobin is less than $100 \mathrm{~g} / \mathrm{L}$ during pregnancy, anemia was defined. Thrombocytopenia was defined the count of platelet less than $100 \times 109 /$ L. When amniotic fluid index was less than $5 \mathrm{~cm}$ or amniotic fluid vertical depth was less than $2 \mathrm{~cm}$, oligohydramnios was diagnosed. PPH was defined as the loss of blood within 24 hours after delivery, with the amount of bleeding was $500 \mathrm{~mL}$ or more.
Fetal outcomes included fetal loss, prematurity, IUGR and asphyxia neonatorum. Fetal loss referred to pregnancy failure due to spontaneous abortion, therapeutic abortion, stillbirth or neonatal death. Preterm birth referred to live birth between 28 weeks and less than 37 gestational weeks. IUGR was defined as a $10 \%$ lower limit for birth weight below the $\mathrm{Cl}$ of the normal gestational weight curve. An Apgar score $>7$ was considered normal, while a score $\leq 7$ should be considered as asphyxia.

\section{Statistical analysis}

Statistical analysis was performed with SPSS version 19.0. The medical data were expressed as means \pm SD or frequencies. The count data were compared using the $\mathrm{x} 2$ test. Comparisons of measurement data were performed using the Student's t-test appropriately. Logistic regression analyses were not only used to compare multiple factors but also calculated the ORs of $95 \% \mathrm{Cls}$ for maternal-infant outcomes. $\mathrm{P}<0.05$ was considered statistically significant. The missing data were processed by list deletion method and chain equation multiple interpolation method.

\section{RESULTS \\ Patient characteristics}

There was a total of 52 patients with 55 gestations in our study, including 26 cases with active SLE and 29 cases with non-active SLE. The average age at diagnosis of SLE was $22.71 \pm 3.81$ years (range, 14 to 31 years). The exhibitions of the general characteristics were displayed in Table 1. Mean ages, body mass index (BMI) and living area were not significantly different between the two cohorts as the same as the mean duration of SLE and the incidence of caesarean section. There were no significant differences in comorbid conditions including diabetes and/or hypertension diagnosed pre-pregnancy between active and non-active SLE patients. The proportion of women who had a history of spontaneous abortion and nulliparity was not significantly different between the two groups.

Most of cases both from active and non-active SLE groups were treated with low-dose glucocorticoids(GC), prednisone dose ranged from 2.5 to $25 \mathrm{mg}$. There were three cases in the active phase treated with immunosuppressants, including azathioprine, tacrolimus and cyclosporine A. Compared to non-active SLE pregnancies, patients with active SLE had a significant lower proportion taking hydroxychloroquine (HCQ) with dose of $200 \mathrm{mg} / \mathrm{d}$ (42.3\% vs 69.0\%, $\mathrm{p}<0.05)$. Aspirin $(100 \mathrm{mg} / \mathrm{d})$ was taken by patients with positive aPL antibodies. Only two patients with HDP in the quiescent phase of SLE used low-molecular-weight-heparin (LMWH) during their pregnancy. 


\begin{tabular}{|c|c|c|c|}
\hline Variables & Active SLE & $\begin{array}{l}\text { Non-active } \\
\text { SLE }\end{array}$ & $\mathbf{P}$ \\
\hline Age $[y r s$, mean $\pm S D]$ & $28.04 \pm 3.97$ & $30.14 \pm 3.94$ & 0.054 \\
\hline BMI $\left[\mathrm{kg} / \mathrm{m}^{2}\right.$ mean $\left.\pm \mathrm{SD}\right]$ & $23.94 \pm 3.39$ & $23.30 \pm 2.79$ & 0.447 \\
\hline Nulliparity, n (\%) & 20 (76.9\%) & $14(48.3 \%)$ & 0.051 \\
\hline \multicolumn{4}{|l|}{ Region, n (\%) } \\
\hline City & $12(46.2 \%)$ & 17 (58.6\%) & 0.423 \\
\hline Rural & $14(53.8 \%)$ & 12 (41.4\%) & \\
\hline $\begin{array}{l}\text { Duration of SLE [years, } \\
\text { mean } \pm S D \text { ] }\end{array}$ & $5.85 \pm 3.66$ & $6.72 \pm 2.87$ & 0.324 \\
\hline \multicolumn{4}{|l|}{$\begin{array}{l}\text { History of spontaneous } \\
\text { abortion, } n(\%)\end{array}$} \\
\hline With & 15 (57.7\%) & $15(51.7 \%)$ & 0.657 \\
\hline Without & $11(42.3 \%)$ & $14(48.3 \%)$ & \\
\hline $\begin{array}{l}\text { Prepregnancy diabetes, } \\
\mathrm{n}(\%)\end{array}$ & $1(3.8 \%)$ & 0 & 0.473 \\
\hline $\begin{array}{l}\text { Prepregnancy } \\
\text { hypertension, } n(\%)\end{array}$ & $4(15.4 \%)$ & $2(6.9 \%)$ & 0.406 \\
\hline Cesarean section, n (\%) & $14(53.8 \%)$ & $20(69.0 \%)$ & 0.279 \\
\hline \multicolumn{4}{|l|}{$\begin{array}{l}\text { Drugs taken at the onset } \\
\text { of pregnancy }\end{array}$} \\
\hline Prednisone, n (\%) & $25(96.2 \%)$ & 26 (89.7\%) & 0.970 \\
\hline Prednisone dose $(\mathrm{mg} / \mathrm{d})$ & $12.88 \pm 6.19$ & $10.26 \pm 4.93$ & 0.086 \\
\hline HCQ, n (\%) & $11(42.3 \%)$ & $20(69.0 \%)$ & 0.022 \\
\hline $\begin{array}{l}\text { Immunosuppressants, } \\
\text { n (\%) }\end{array}$ & $3(11.5 \%)$ & 0 & 0.099 \\
\hline Aspirin, $n(\%)$ & $7(26.9 \%)$ & $5(17.2 \%)$ & 0.238 \\
\hline LMWH, n (\%) & 0 & $2(6.9 \%)$ & 0.173 \\
\hline
\end{tabular}

SLE — systemic lupus erythematosus; SD — standard deviation; BMI — body mass index; $\mathrm{HCQ}$ - hydroxychloroquine; LMWH — low molecular weight heparin

\section{Comparison of laboratory indexes of SLE in different stages}

In the present study, significant differences in autoantibodies were observed between the active and non-active SLE groups, including anti-ds DNA ( $53.8 \%$ vs $24.1 \%)$, anti-Sm (50.0\% vs $24.1 \%$ ), anti-Ro/SSA ( $84.6 \%$ vs $58.6 \%$ ). Compared to non-active SLE pregnancies, patients with active SLE had significant hyperimmunoglobulin ( $57.7 \%$ vs $20.7 \%)$,but had hypocomplement (76.9\% vs $24.1 \%$ ). Low PLT, high ESR, CRP and 24-h PRO during pregnancy were significantly associated with SLE activity. No other significant differences were found in the laboratory findings between the two cohorts (Tab. 2).

\section{Risk factors affecting fetal loss}

Based on the results of single factor analyses, fetal loss was correlated with presence of taking hydroxychloroquine (HCQ), positive rate of anti-ds DNA, anti-Sm and anti-Ro/SSA, hyperimmunoglobulin, hypocomplement, low PLT, high
Table 2. Comparison of laboratory indexes of systemic lupus erythematosus (SLE) patients in different states

\begin{tabular}{|c|c|c|c|}
\hline Variables & Active SLE & $\begin{array}{l}\text { Non-active } \\
\text { SLE }\end{array}$ & $\mathbf{P}$ \\
\hline ANA, n (\%) & $26(100.0 \%)$ & 28 (96.6\%) & 0.339 \\
\hline anti-dsDNA, n (\%) & $14(53.8 \%)$ & 7 (24.1\%) & 0.024 \\
\hline anti-Sm n (\%) & $13(50.0 \%)$ & $7(24.1 \%)$ & 0.047 \\
\hline anti-Ro/SSA, n (\%) & $22(84.6 \%)$ & $17(58.6 \%)$ & 0.034 \\
\hline anti-La/SSB, n (\%) & 7 (26.9\%) & $4(13.8 \%)$ & 0.224 \\
\hline aPL, n (\%) & $10(38.5 \%)$ & $5(17.2 \%)$ & 0.129 \\
\hline $\begin{array}{l}\text { Hyperimmunoglobulin, } \\
\mathrm{n}(\%)\end{array}$ & $15(57.7 \%)$ & $6(20.7 \%)$ & 0.006 \\
\hline$A, n(\%)$ & $9(34.6 \%)$ & $2(6.9 \%)$ & 0.017 \\
\hline$M, n(\%)$ & $8(30.8 \%)$ & $2(6.9 \%)$ & 0.035 \\
\hline $\mathrm{G}, \mathrm{n}(\%)$ & 11 (42.3\%) & $3(10.3 \%)$ & 0.012 \\
\hline $\begin{array}{l}\text { Hypocomplement, } \\
\mathrm{n}(\%)\end{array}$ & $20(76.9 \%)$ & $7(24.1 \%)$ & $<0.001$ \\
\hline C3, n (\%) & $17(65.4 \%)$ & $6(20.7 \%)$ & 0.001 \\
\hline C4, n (\%) & $16(61.5 \%)$ & $7(24.1 \%)$ & 0.005 \\
\hline $\mathrm{HB}[\mathrm{g} / \mathrm{L}]$ & $109.15 \pm 12.66$ & $111.17 \pm 16.95$ & 0.622 \\
\hline WBC $[\times 109 / L]$ & $8.06 \pm 2.79$ & $9.32 \pm 3.58$ & 0.155 \\
\hline PLT [x109/L] & $125.23 \pm 51.20$ & $197.59 \pm 61.57$ & $<0.001$ \\
\hline $\mathrm{ESR}[\mathrm{mm} / \mathrm{h}]$ & $32.10 \pm 13.51$ & $20.24 \pm 8.73$ & $<0.001$ \\
\hline CRP [mg/L] & $15.00 \pm 13.76$ & $8.69 \pm 8.92$ & 0.045 \\
\hline PRO, n (\%) & $16(61.5 \%)$ & $12(41.4 \%)$ & 0.180 \\
\hline 24-h PRO [g/24 h] & $0.92 \pm 1.16$ & $0.34 \pm 0.24$ & $<0.001$ \\
\hline$A L B[g / L]$ & $30.65 \pm 2.88$ & $32.81 \pm 2.76$ & 0.701 \\
\hline CR [umol/L] & $52.62 \pm 13.99$ & $49.79 \pm 10.78$ & 0.157 \\
\hline BUN [mmol/L] & $3.80 \pm 1.10$ & $3.54 \pm 1.38$ & 0.305 \\
\hline $\mathrm{TC}[\mathrm{mmol} / \mathrm{L}]$ & $3.48 \pm 1.12$ & $3.04 \pm 0.72$ & 0.082 \\
\hline $\mathrm{TG}[\mathrm{mmol} / \mathrm{L}]$ & $4.90 \pm 0.97$ & $4.43 \pm 0.85$ & 0.063 \\
\hline LDL-C [mmol/L] & $2.55 \pm 0.55$ & $2.49 \pm 1.00$ & 0.760 \\
\hline $\mathrm{HDL}-\mathrm{C}[\mathrm{mmol} / \mathrm{L}]$ & $1.47 \pm 0.38$ & $1.55 \pm 0.38$ & 0.399 \\
\hline
\end{tabular}

SLE — systemic lupus erythematosus; ANA — antinuclear antibodies; HB - hemoglobin; WBC — white blood cell; PLT — platelet; ESR - erythrocyte sedimentation rate; $\mathrm{CRP}$ - C-reactive protein; $\mathrm{PRO}$ - urine protein; 24-h PRO - 24-hour urine protein; ALB - albumin; CR - serum creatinine; BUN — blood urea nitrogen; $\mathrm{TC}$ - totalcholesterol; TG — triglyceride

ESR, CRP and 24h PRO. As show in Table 3, when factors related to fetal loss in univariate analysis were used as independent variables in multivariate logistic regression analysis, it was found that low PLT, hypocomplement and high 24-h PRO were significantly correlated with fetal loss.

\section{Pregnancy outcomes}

HDP was diagnosed in $50.0 \%$ of the active SLE patients. The risk of HDP (OR $4.588,95 \% \mathrm{Cl} 1.084$ to 19.416) remained high after adjusting for confounding factors. The incidence of thrombocytopenia and lupus nephritis in women with active SLE was significantly higher than those in the 


\begin{tabular}{|l|l|l|l|l|l|l|l|l|l|}
\hline Table 3. Multiple factor logistic regression analysis for Risk factors affecting fetal loss \\
\hline Variables & B & SE & Wald & DF & p value & OR & \multicolumn{2}{|l|}{$\mathbf{9 5 \% ~ C l ~ o f ~ O R ~}$} \\
\hline 24-h urine protein & -5.635 & 2.573 & 4.797 & 1 & 0.029 & 0.004 & 0.000 & 0.553 \\
\hline Platelet counts & 0.029 & 0.014 & 4.082 & 1 & 0.043 & 1.029 & 1.001 & 1.059 \\
\hline Hypocomplement C3/C4 & 5.122 & 2.291 & 5.000 & 1 & 0.025 & 6.795 & 1.882 & 14.938 \\
\hline
\end{tabular}

$\mathrm{SE}$ - standard error; $\mathrm{DF}$ — degrees of freedom; $\mathrm{Cl}$ — confidence interval; $\mathrm{OR}$ — odds ratio; $\mathrm{B}$ - regression coefficient

Table 4. Comparison of pregnancy outcomes between active SLE and inactive SLE patients

\begin{tabular}{|c|c|c|c|c|}
\hline Pregnancy outcome & Active SLE & Non-active SLE & $\mathbf{P}$ & OR $(95 \% \mathrm{Cl})$ \\
\hline \multicolumn{5}{|l|}{ Maternal outcome } \\
\hline HDP & $13(50.0 \%)$ & $6(20.7 \%)$ & 0.048 & 4.588 (1.084-19.416) \\
\hline GDM & $1(3.8 \%)$ & $1(3.4 \%)$ & 0.937 & $1.120(0.067-18.861)$ \\
\hline Anemia & 7 (26.9\%) & $7(24.1 \%)$ & 0.813 & $1.158(0.344-3.899)$ \\
\hline Thrombocytopenia & $7(26.9 \%)$ & $1(3.4 \%)$ & 0.014 & $10.316(1.172-90.780)$ \\
\hline Lupus nephritis & $4(15.4 \%)$ & 0 & 0.028 & $1.846(0.718-4.997)$ \\
\hline PROM & $11(42.3 \%)$ & $9(31.0 \%)$ & 0.415 & $1.630(0.539-4.927)$ \\
\hline Oligohydramnios & $6(23.1 \%)$ & $5(17.2 \%)$ & 0.739 & $1.440(0.382-5.428)$ \\
\hline $\mathrm{PIH}$ & $3(11.5 \%)$ & $2(6.9 \%)$ & 0.550 & $1.761(0.270-11.467)$ \\
\hline Placental infarction & $15(57.7 \%)$ & $8(27.6 \%)$ & 0.031 & $3.580(1.161-11.040)$ \\
\hline Chorioamnionitis & $11(42.3 \%)$ & $14(48.3 \%)$ & 0.788 & $0.786(0.271-2.281)$ \\
\hline \multicolumn{5}{|l|}{ Fetal outcome } \\
\hline Fetal loss & $5(19.2 \%)$ & 0 & 0.019 & $1.808(0.670-1.970)$ \\
\hline Premature & $11(42.3 \%)$ & $6(20.7 \%)$ & 0.038 & $3.833(1.124-13.076)$ \\
\hline IUGR & $6(23.1 \%)$ & $4(13.8 \%)$ & 0.295 & $2.344(0.571-9.621)$ \\
\hline Asphyxia neonatorum & $7(26.9 \%)$ & $2(6.9 \%)$ & 0.029 & $6.300(1.158-34.262)$ \\
\hline
\end{tabular}

other cohort, and the risks of thrombocytopenia (OR 10.316, $95 \% \mathrm{Cl} 1.172$ to 90.780 ) and lupus nephritis (OR 1.846, 95\% $\mathrm{Cl} 0.718$ to 4.997$)$ remained unchanged after adjusting for confounding factors. Placental infarction was commonly founded among active SLE patients, and the rate was 3.58fold higher in the group of active SLE than that in non-active SLE. Significant differences in the remaining manifestations of maternal outcomes, including GDM, anemia, PROM, oligohydramnios, PPH and chorioamnionitis, were not observed between the two groups. Peripartum infection was not occurred in two groups, except one SLE patient in the quiescent phase. Maternal deaths did not occur in either groups.

Fetal loss occurred in four cases in the active SLE phase. Two cases were accompanied by lupus nephritis (one case with intrauterine fetal death and the other with neonatal death). The rest of two cases with therapeutic abortion were in relation to immunosuppressant therapy. After adjusting for confounding factors, the total fetal loss rate (OR 1.808, $95 \% \mathrm{Cl} 0.670-1.974$ ) of patients with active SLE was higher than that of patients with inactive SLE. In the two cohorts, preterm births accounted for $42.3 \%$ and $20.7 \%$, respectively.
The adjusted OR remained high for outcome (OR 3.833, 95\% $\mathrm{Cl} 1.124$ to 13.076). Furthermore, we also observed higher rate of asphyxia neonatorum for pregnant women with disease activity. The Apgar score (1 $\mathrm{min}$ ) in active SLE group was remarkably lower than that of the other group. It was observed in $26.9 \%$ and $6.9 \%$ of pregnancies, respectively, in the two cohorts. When the confounding factors were adjusted, the risk of asphyxia neonatorum (OR 6.300, 95\% Cl 1.158 to 34.262 was still high. Finally, significant difference in the rate of IUGR was not observed between the two cohorts (Tab. 4).

\section{DISCUSSION}

Most of studies show the increased flare rates by $25-65 \%$ $[7,9,10]$. Other researches have evaluated different outcomes of lupus flares during pregnancy and shown a low incidence of flare (19.4-25\%). In this study, 26 (47.3\%) of the patients experienced SLE activity during pregnancy. Most of the disease activity observed in these patients ranged from mild to moderate, with new skin-joint changes. The active period of the disease mainly occurred after the second trimester. 
In order to avoid adverse maternal and fetal outcomes during pregnancy, rational and standardized drug treatment was very important. GC, especially prednisone, had been the hallmark medication for SLE and were especially the more widely used therapy to control mild-severe flares of SLE [14]. However, during pregnancy, drug dose should be minimised as much as possible, high dose should be taken in a short time during disease flare, and drug dose must be administered during delivery $[2,15]$. In general, the basis for the use of different dosages of GC in a specific clinical setting in SLE is essentially empirical. In our sample, 25 (96.2\%) pregnant women with SLE in active phase and 26 (89.7\%) patients in non-active phase were treated with low-dose prednisone dose, ranged from 2.5 to $25 \mathrm{mg}$.

HCQ was shown to reduce the rates of lupus fares in pregnancy and was widely applied in the treatment of SLE in recent years and has a good reputation in controlling skin complications from SLE $[11,16]$. Our prevalence usage of $\mathrm{HCQ}$ was $42.3 \%$ in active phase and $69 \%$ in non-active phase, which was higher than that prescribed from $18 \%$ to $32.2 \%$ in Spain [17],but close to $55 \%$ in the US [18]. The possible reasons listed as follows: Firstly, our patients had the scope from Jan 2007 to Jan 2019. Although on the early years, HCQ was not used in our country extensively, case data were mainly concentrated in the past 10 years with HCQ used widely. Secondly, in China, when patients with SLE entered the stage of fertility, they were advised to accept management not only from obstetricians but also from rheumatologists, who would prescrib HCQ more frequently.

Durcan et al. [19] found the SLE patients had abnormal lipoproteins, including a decrease in HDL and an increase in LDL, and suggested that HDL and LDL can be used as one of the important parameters to evaluate the disease activity. In our study, there was no obvious difference in lipid and lipoproteins between two groups. This finding might be due to the less cases in our study. In the follow-up study, we will conduct a multicenter study and bring more cases into our groups to obtain more data.

The caesarean section surgery was accepted by 34 (61.8\%) patients in our study. The absolute rate was surprisingly high in both groups, and it was much higher than that reported in most previous studies, but lower than that of another Chinese study [15,20]. SLE was still a risk factor for maternal and fetal outcomes. Most SLE women were inclined to undergo cesarean to terminate pregnancy, which was mainly because doctors and patients preferred elective cesarean to prevent complications closely related to SLE during birth.

Multivariable analysis showed that fetal loss correlated with low PLT, hypocomplement and $24 \mathrm{~h}$ PRO in the present study, which was in line with the previous reports [21, 22]. It directly confirmed the adverse effect of SLE activity on fetal outcome. The results of our study confirmed that almost one third of SLE pregnant women had obvious HDP complications, diagnosed in $50.0 \%$ of the women with active SLE and $20.7 \%$ of the women with non-active SLE. The OR remained significant, even after adjusting for confounding factors. It was in line with the reported literatures [9, 23]. Previous studies demonstrated that thrombocytopenia and lupus nephritis were significantly associated with adversely maternal-infatal outcomes [23-25]. Based on our findings, it was demonstrated as important risk factors for severe obstetric outcomes.

Additionally, placental infarction occurred in nearly $42 \%$ pregnant women with SLE, and especially 3.58-fold higher in the patients with active SLE than the others. This finding was consistent with the result described in the study Magid MS et al. [26]. Some research mainly focused on the risk factors of poor fetal outcomes in different phases of SLE. Zhan et al. [27] also carried on a retrospective study of 263 pregnant patients with SLE, which showed that patients with active lupus had a 12.4-fold higher risk of fetal loss. Moreover, Ku et al. [28] discovered an association between active disease and live births. In our study, the total fetal loss rate was higher in the active SLE patients (OR 1.808, 95\% Cl 0.670 to 1.974 , which was lower than the previous reports and probably due to the more use of GC and HCQ. Furthermore, in our study, a higher rate of preterm and asphyxia neonatorum was observed in the active SLE group (42.3\% and $26.9 \%$, respectively). The adjusted $O R$ remained high for outcomes ( $O R=3.833$ and $O R=6.300$, respectively). The results was similar to several other studies $[29,30]$.

\section{Limitations}

There were several limitations to our study. Firstly, we had some data loss because it was a retrospective study. Secondly, patients were usually referred to our institution after the second trimester and lacked information at the pre and beginning of pregnancy. So, in our work, there were no cases in the early abortion, whether spontaneous or therapeutic. Thirdly, our single-center study might have had bias. Additionally, most patients with SLE during pregnancy did not have postpartum visits in our hospital. Finally, since many patients entered this study only after pregnancy, we did not have information to review the activity of the disease before conception and to define whether conception increased the risk of activity or not.

\section{CONCLUSIONS}

In conclusion, the risks of HDP, thrombocytopenia, lupus nephritis and placental infarction increase significantly in active SLE women during pregnancy. In addition, SLE in active stage leads to higher rates of fetal loss, premature delivery and asphyxia neonatorum. Therefore, we suggest 
that when these patients enter the stage of fertility, particularly in active period, they are still considered to have high-risk pregnancies, should have full access to preconception counselling, and should be ideally managed under the coordinated care of obstetricians, rheumatologists and other specialists as needed.

\section{Acknowledgements}

The authors would like to thank Ms. Xueqi Guo and Xiaohong Lan from the hospital of case collection for coordinating the research and assistance in data arrangement.

\section{Conflict of interest}

The authors declare that they have no competing interests.

\section{REFERENCES}

1. Draborg AH, Duus K, Houen G. Epstein-Barr virus and systemic lupus erythematosus. Clin Dev Immunol. 2012; 2012: 370516, doi: 10.1155/2012/370516, indexed in Pubmed: 22811739.

2. Lateef A, Petri M. Systemic Lupus Erythematosus and Pregnancy. Rheum Dis Clin North Am. 2017; 43(2): 215-226, doi: 10.1016/j.rdc.2016.12.009, indexed in Pubmed: 28390564.

3. Moroni G, Ponticelli C, Ponticelli C, et al. The risk of pregnancy in patients with lupus nephritis. J Nephrol. 2003; 16(2): 161-167, indexed in Pubmed: 12768062.

4. KalokA, Abdul Cader R, Indirayani I, et al. Pregnancy outcomes in systemic lupus erythematosus (SLE) women. Horm Mol Biol Clin Investig. 2019; 40(3), doi: 10.1515/hmbci-2019-0007, indexed in Pubmed: 31553696.

5. Zamani B, Shayestehpour M, Esfahanian F, et al. The study of factors associated with pregnancy outcomes in patients with systemic lupus erythematosus. BMC Res Notes. 2020; 13(1): 185, doi: 10.1186/s13104020-05039-9, indexed in Pubmed: 32228711.

6. de Jesus GR, Mendoza-Pinto C, de Jesus NR, et al. Understanding and Managing Pregnancy in Patients with Lupus. Autoimmune Dis. 2015; 2015: 943490, doi: 10.1155/2015/943490, indexed in Pubmed: 26246905.

7. Davis-Porada J, Kim MY, Guerra MM, et al. Low frequency of flares during pregnancy and post-partum in stable lupus patients. Arthritis Res Ther. 2020; 22(1): 52, doi: 10.1186/s13075-020-2139-9, indexed in Pubmed: 32188491.

8. Touma Z, Urowitz MB, Taghavi-Zadeh S, et al. Systemic lupus erythematosus disease activity index 2000. J Rheumatol. 2002; 29(2): 288-291, indexed in Pubmed: 11838846.

9. Lateef A, Petri M. Systemic Lupus Erythematosus and Pregnancy. Rheum Dis Clin North Am. 2017; 43(2): 215-226, doi: 10.1016/j.rdc.2016.12.009, indexed in Pubmed: 28390564.

10. Eudy AM, Siega-Riz AM, Engel SM, et al. Efect of pregnancy on disease fares in patients with systemic lupus erythematosus. Ann Rheum Dis. 2018; 77: 855-860.

11. Götestam Skorpen C, Lydersen S, Gilboe IM, et al. Disease Activity During Pregnancy and the First Year Postpartum in Women With Systemic Lupus Erythematosus. Arthritis Care Res (Hoboken). 2017; 69(8): 1201-1208, doi: 10.1002/acr.23102, indexed in Pubmed: 27696790.

12. Lockshin MD. Pregnancy does not cause systemic lupus erythematosus to worsen. Arthritis Rheum. 1989; 32(6): 665-670, doi: 10.1002/anr.1780320602, indexed in Pubmed: 2638570.

13. Cavallasca JA, Laborde HA, Ruda-Vega $H$, et al. Maternal and fetal outcomes of 72 pregnancies in Argentine patients with systemic lupus erythematosus (SLE). Clin Rheumatol. 2008; 27(1): 41-46, doi: 10.1007/s10067-007-0649-3, indexed in Pubmed: 17516127.
14. Magro-Checa C, Zirkzee EJ, Huizinga TW, et al. Management of Neuropsychiatric Systemic Lupus Erythematosus: Current Approaches and Future Perspectives. Drugs. 2016; 76(4): 459-483, doi: 10.1007/s40265015-0534-3, indexed in Pubmed: 26809245.

15. Wu J, Ma J, Bao C, et al. Pregnancy outcomes among Chinese women with and without systemic lupus erythematosus: a retrospective cohort study. BMJ Open. 2018; 8(4): e020909, doi: 10.1136/bmjopen-2017-020909, indexed in Pubmed: 29654043.

16. Eudy AM, Siega-Riz AM, Engel SM, et al. Effect of pregnancy on disease flares in patients with systemic lupus erythematosus. Ann Rheum Dis. 2018; 77(6): 855-860, doi: 10.1136/annrheumdis-2017-212535, indexed in Pubmed: 29463519.

17. Molina MJ, Mayor AM, Franco AE, et al. Utilization of health services and prescription patterns among lupus patients followed by primary care physicians and rheumatologists in Puerto Rico. Ethn Dis. 2008; 18(2 Suppl 2): S2-S205, indexed in Pubmed: 18646350.

18. Schmajuk G, Yazdany J, Trupin L, et al. Hydroxychloroquine treatment in a community-based cohort of patients with systemic lupus erythematosus. Arthritis Care Res (Hoboken). 2010; 62(3): 386-392, doi: 10.1002/acr.20002, indexed in Pubmed: 20391485.

19. Durcan L, Winegar DA, Connelly MA, et al. Longitudinal Evaluation of Lipoprotein Variables in Systemic Lupus Erythematosus Reveals Adverse Changes with Disease Activity and Prednisone and More Favorable Profiles with Hydroxychloroquine Therapy. J Rheumatol. 2016; 43(4): 745-750, doi: 10.3899/jrheum.150437, indexed in Pubmed: 26834214.

20. HeWR, Wei H. Maternal and fetal complications associated with systemic lupus erythematosus: An updated meta-analysis of the most recent studies (2017-2019). Medicine (Baltimore). 2020; 99(16): e19797, doi: 10.1097/MD.0000000000019797, indexed in Pubmed: 32311994.

21. Buyon JP, Kim MY, Guerra MM, et al. Predictors of Pregnancy Outcomes in Patients With Lupus: A Cohort Study. Ann Intern Med. 2015; 163(3): 153-163, doi: 10.7326/M14-2235, indexed in Pubmed: 26098843.

22. Moroni G, Doria A, Giglio E, et al. Fetal outcome and recommendations of pregnancies in lupus nephritis in the 21 st century. A prospective multicenter study. J Autoimmun. 2016; 74: 6-12, doi: 10.1016/j. jaut.2016.07.010, indexed in Pubmed: 27496151.

23. Chen D, Lao M, Zhang J, et al. Fetal and Maternal Outcomes of Planned Pregnancy in Patients with Systemic Lupus Erythematosus: A Retrospective Multicenter Study. J Immunol Res. 2018; 2018: 2413637, doi: 10.1155/2018/2413637, indexed in Pubmed: 30255104.

24. Wagner SJ, Craici I, Reed D, et al. Maternal and foetal outcomes in pregnant patients with active lupus nephritis. Lupus. 2009; 18(4): 342-347, doi: 10.1177/0961203308097575, indexed in Pubmed: 19276302.

25. Saavedra MA, Cruz-Reyes C, Vera-Lastra O, et al. Impact of previous lupus nephritis on maternal and fetal outcomes during pregnancy. Clin Rheumatol. 2012;31(5):813-819, doi: 10.1007/s10067-012-1941-4, indexed in Pubmed: 22278163.

26. Magid MS, Kaplan C, Sammaritano LR, et al. Placental pathology in systemic lupus erythematosus: a prospective study. Am J Obstet Gynecol. 1998; 179(1): 226-234, doi: 10.1016/s0002-9378(98)70277-7, indexed in Pubmed: 9704792.

27. Zhan Z, Yang $Y$, Zhan $Y$, et al. Fetal outcomes and associated factors of adverse outcomes of pregnancy in southern Chinese women with systemic lupus erythematosus. PLoS One. 2017; 12(4): e0176457, doi: 10.1371/journal.pone.0176457, indexed in Pubmed: 28441446.

28. Ku M, Guo S, Shang W, et al. Pregnancy Outcomes in Chinese Patients with Systemic Lupus Erythematosus (SLE): A Retrospective Study of 109 Pregnancies. PLoS One. 2016; 11(7): e0159364, doi: 10.1371/journal. pone.0159364, indexed in Pubmed: 27442513.

29. Wallenius $M$, Salvesen $K \AA ̊$, Daltveit AK, et al. Systemic lupus erythematosus and outcomes in first and subsequent births based on data from a national birth registry. Arthritis Care Res (Hoboken). 2014; 66(11): 1718-1724, doi: 10.1002/acr.22373, indexed in Pubmed: 24839126.

30. Naseri EP, Surita FG, Borovac-Pinheiro A, et al. Systemic Lupus Erythematosus and Pregnancy: A Single-Center Observational Study of 69 Pregnancies. Rev Bras Ginecol Obstet. 2018; 40(10): 587-592, doi: 10.1055/s-0038-1672136, indexed in Pubmed: 30352455. 